\title{
Shape vision in bees: innate preference for flower-like patterns
}

\author{
M. LEHRER*, G. A. HORRIDGE, S. W. ZHANG AND R. GADAGKAR§
}

Centre for Visual Sciences, Research School of Biological Sciences, The Australian National University, P.O. Box 475, Canberra ACT 2601, Australia

\section{SUMMARY}

The bees' spontaneous preferences toward various black-and-white patterns were studied using a multiple-choice test procedure. The patterns are presented on vertical planes, and the bees' choices at a fixed distance from the patterns are recorded. To exclude a possible influence of the bees' previous experience with natural flowers, the bees are trained to randomized checkerboard patterns prior to testing them with sets of other patterns. We find that, when the test patterns are of the same kind, but differ in their spatial frequencies, the bees prefer low over high frequencies. However, when the patterns differ in type, the bees express, regardless of spatial frequency, a positive preference for patterns containing radiating elements, and a negative preference for patterns containing circular elements or elements arranged at random. We find, in addition, that symmetrical patterns are more attractive than less symmetrical or non-symmetrical patterns. We propose that bees respond innately to some features of natural flowers, resulting in a spontaneous preference for radiating, as well as symmetrical patterns.

\section{INTRODUGTION}

Shape perception in honeybees has been examined for many decades by one of two different experimental approaches. The majority of the studies (reviewed by Wehner 1981) exploited the bee's excellent learning capacity, i.e. bees were trained to collect sugar water from a particular pattern, and were then tested by giving them a choice between that pattern and others that differed from the trained pattern in one parameter or another. The earlier training experiments were conducted using patterns presented on a horizontal plane, and the criterion for choosing a pattern was the bees landing on it. This procedure suffers from two major disadvantages. First, when a pattern is presented on a horizontal plane, its appearance depends on the direction from which the bee arrives. The bee is therefore unable to store any particular idetic (photographic) image of the pattern, nor any particular orientation of contours, and can use only spaceinvariant cues. Secondly, at the instant of landing (which was the criterion for the bee's choice), the bee is too near to the pattern to perceive its global appearance, and can use only local cues.

The results of these studies indeed show that, under these conditions, the bees do not discriminate between closed shapes such as squares and triangles (von Frisch 1915; Hertz 1930; Manning 1957), but they dis-

* Present address: Zoological Institute, Department of Neurobiology, University of Zurich, Winterthurerstrasse 190, CH-8057 Zurich, Switzerland.

$\S$ Present address: Centre for Ecological Sciences, Indian Institute of Science, Bangalore-560 012, India. criminate very well between patterns that differ in their degree of disruption (contour density) (von Frisch 1915; Hertz 1930; Zerrahn 1934). The principal parameter used in pattern discrimination was found to be the amount of contour per unit area of the pattern, and thus the pattern's spatial frequency (Zerrahn 1934; von Frisch 1965).

The second method for investigating pattern discrimination is to look at the insect's spontaneous preferences for various pattern parameters. Measuring spontaneous preferences is a very useful method for studying the performance of animals that cannot be trained to a particular stimulus, such as flies (Reichardt 1973), crickets (Campan \& Lacoste 1971), and beetles (Varjú 1976; Dafni 1991) (for further references, see Wehner 1981, Table 4). In the bee, which can easily be trained to associate a particular stimulus with a food reward, investigations involving spontaneous preferences have been conducted less frequently than training experiments. The usual procedure was to train bees to collect sugar water at a site that was not associated with any particular pattern, and then give them, at the same site, a choice among various patterns that were all novel to the bees (Hertz 1930; Free 1970; Anderson 1977). The patterns were, again, presented on a horizontal plane, with landing as the choice criterion, and thus the same problems arise as in the discrimination experiments mentioned above. In addition, 'spontaneous' pattern preferences measured in this way are not neccessarily truly spontaneous, because the bees' previous experience with natural flowers (that cannot be controlled) might have already led to learning particular pattern parameters, reflected 
in the later tests. Nonetheless, experiments using the two different methods have rendered complementary results. It was shown that a spontaneous preference toward a particular pattern parameter leads to a better and faster learning of that parameter in training experiments, and that patterns that have previously been shown to be unattractive are hardly learned even during a long training (von Frisch 1915; Hertz 1930; Zerrahn 1934). In all of these studies, bees were shown to spontaneously prefer disrupted patterns (patterns of high spatial frequency) over less disrupted ones, which was in agreement with the results of the training experiments. The experimental data thus validated the so-called flicker-theory of shape perception already proposed by Exner (1876), based on the anatomy of the compound eye. This theory says that pattern discrimination in insects is governed by temporal rather than spatial parameters. When the bee approaches the pattern and lands on it, a larger amount of contours per unit area of the pattern produces a higher flicker frequency (of 'on' and 'off' stimulation) at the bee's eye than do coarser patterns.

The flicker theory of insect vision persisted for several decades. In his famous monograph, Karl von Frisch $(1965$, p. 494) explains the bees' performance in pattern discrimination tasks by saying: "The mere spatial proximity is not of such great consequence as for us, but rather the continually changing pattern of the alternation of light and darkness' (the author's own translation of the German text). Even in the seventies it was still argued that 'the order of attractiveness of patterns can be predicted from the smallest flicker interval and the number of such intervals (the frequency of encountering them).' (Anderson 1977, p. 86). We note that the data show that this is true for landing bees.

Although the flicker theory was very adequate to explain the early results obtained with patterns presented on a horizontal plane, it could not account for the results of later studies conducted by Wehner using patterns presented on a vertical plane. In Wehner's experimental setup (first introduced by Wehner \& Lindauer 1966), the bee could approach the pattern from only one well-defined direction, and thus the spatial relation between the bee's eye and the pattern was fairly constant. Using this method, Wehner showed that bees store an idetic (i.e. photographic, template-like) image of the pattern (Wehner 1972a, 1981 ; see also Gould 1986), and discriminate well even between patterns that do not differ in their spatial frequency, but rather in other parameters, such as the orientation of contours (Wehner 1972b), the overlap of areas (Wehner 1972 $a$; see also Cruse 1972), or their position in the visual field (Wehner 1972a, $b, 1981$ ). Wehner's criterion for the bees' choices was the bees landing on the opening of a tube in the centre of the pattern.

Over the last few years, a number of studies conducted in Canberra, again using patterns presented on vertical planes, employed an Y-maze setup, first introduced by Srinivasan \& Lehrer (1988), that enables to measure the bees' choices between two patterns viewed at some distance, i.e. before the bee has had a chance to approach one of the patterns and land on it. Using this setup in training experiments, it was shown that, for pattern discrimination at some distance, bees use global, rather than local features of the pattern (Zhang et al. 1992). In most of this work, the spatial orientation of contours was the only parameter offered to the bees. It was shown that the predominant orientation of contours, irrespective of pattern, is used efficiently in pattern discrimination tasks (Van Hateren et al. 1990; Srinivasan et al. 1993; Srinivasan et al. 1994).

The experiments described in the present study were conducted using a modified version of the Y-maze, with 12 compartments, rather than two. Thus, our experiments involve multiple-choice, rather than dualchoice tests. Multiple-choice tests have the advantage that a particular parameter can be varied along a continuum in a single test, or several parameters can be tested simultaneously, and thus the bees' responses to them can be compared directly. Multiple-choice tests have already been employed in earlier studies on the bees' spontaneous pattern preferences (Hertz 1930; Anderson 1977). Our experiments differ, however, from the earlier studies in four respects: (i) we use patterns placed on vertical planes, thus expecting the bees' spontaneous preferences to be directed toward spatial rather than temporal parameters; (ii) we measure the choices made at some distance from the pattern, thus testing global rather than local parameters; (iii) we use patterns with no predominant or average orientation of contours, and thus this parameter cannot be used as a cue; and (iv) prior to testing the bees with sets of novel patterns, we train them to a set of particular patterns (see below), rather than to a site where no pattern is present, thus eliminating the possibility that the bees might use previous experience with natural flowers in the task. It has already been shown that bees trained to a new colour (Menzel 1969) or a new shape (Lehrer, unpublished results) 'forget' the colour or shape they have learned during a previous training. In subsequent discrimination tests, they use only the parameter they have learned recently, and not the one they had learned in the preceding training.

The patterns we use for training the bees are checkerboards, i.e. patterns that do not resemble natural flowers in any respect. In addition, to act against the bees' spontaneous preference for highfrequency patterns (see above), we randomize the spatial frequency of the checkerboard patterns throughout the training, i.e. the bees are trained to a mixture of fine and coarse patterns. We randomize, in addition, the orientation of contours contained in the checkerboard patterns, because this is another parameter that bees have been shown to use in pattern discrimination tasks (see above). The method of randomizing a particular parameter and thus forcing the bees to use a different one has already been successfully employed in experiments investigating the use of motion cues, rather than size cues, in the task of distance estimation (Lehrer et al. 1988; Srinivasan et al. 1989). Thus, in the present study we are as near as possible to looking at the bees' truly spontaneous pattern preferences. 
(a)

(b)
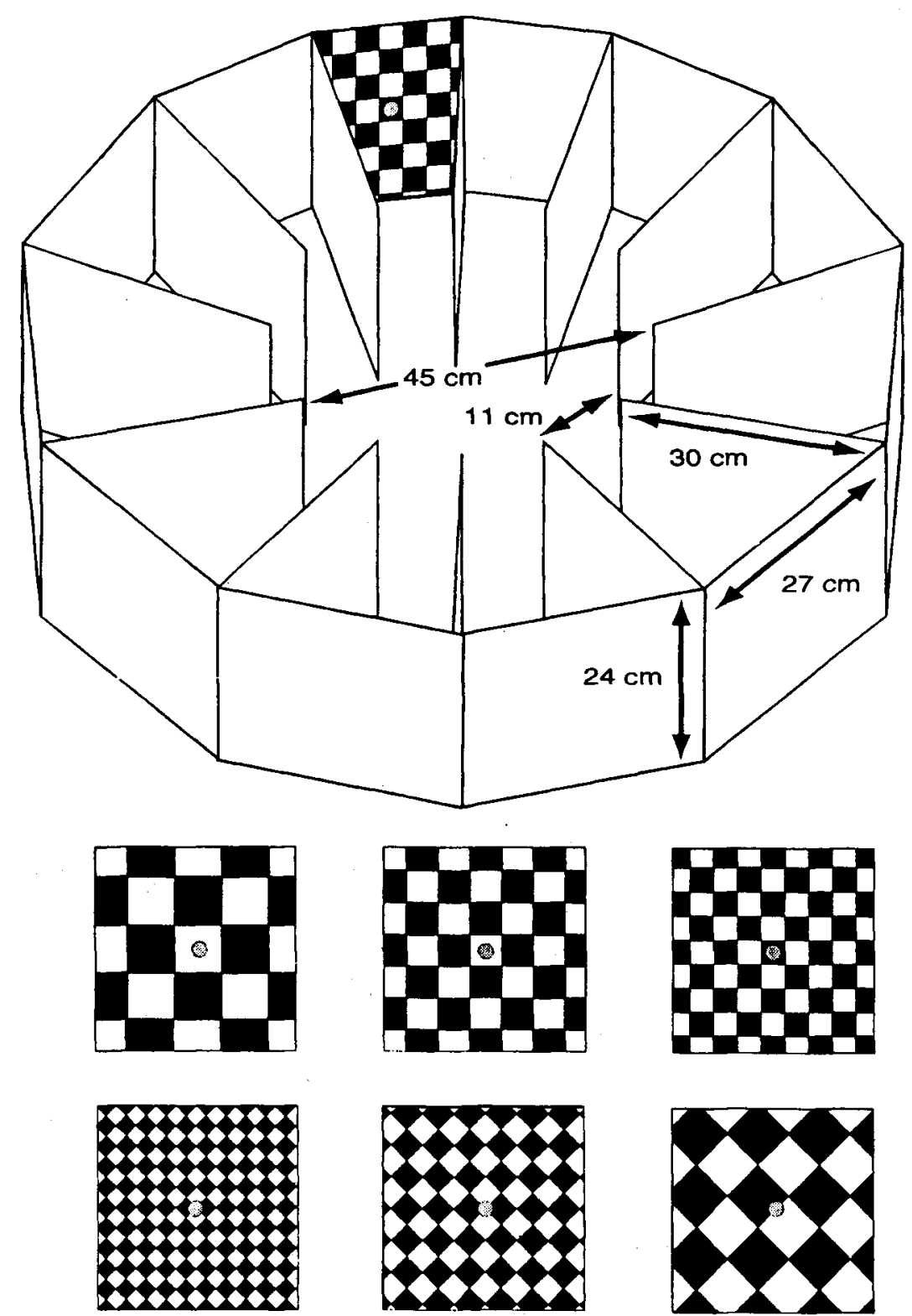

Figure 1. (a) General view of the experimental apparatus in the training situation. One of the 12 compartments has a checkerboard pattern placed on the back wall. A feeder containing sucrose solution was placed in a dark box behind the pattern. The access to the feeder was through a tube in the centre of the pattern. The other 11 compartments displayed white paper and offered no reward. The apparatus rested on a turntable and was rotated about its centre at regular intervals. (b) The six checkerboard patterns used in a semi-random succession during training and between tests. In the tests, with no reward present, each of the 12 compartments had a pattern placed on its back wall, as specified in the text. The criterion for a choice was the bees entering a compartment.

\section{METHODS}

The experimental apparatus (figure $1 a$ ) was placed outdoors in the garden of the Centre for Visual Sciences in Canberra, about $20 \mathrm{~m}$ from the hive from which the experimental bees were recruited. To protect it from direct sunlight, the apparatus was placed under a roof and was screened against south, east and west. This measure was taken to ensure that no conspicuous shadows were present within the apparatus that could have influenced the bees' preferences (see Hoefer \& Lindauer 1976).

The apparatus consisted of 12 compartments opening upon a central circular arena. In the centre of the back wall of each compartment there was a hole (20 $\mathrm{mm}$ in diameter) with a short plexiglass tube penetrating it, protruding about $10 \mathrm{~mm}$ to both sides (front and back) of the wall. Each of the patterns used had a similar hole in its centre and could thus be mounted on the tube in any of the 12 compartments. During training, 11 compartments each displayed a plain white disc $(24 \mathrm{~cm}$ in diameter). The tubes associated with these compartments were closed from the back by means of black paper. The twelfth compartment had a black-and-white checkerboard pattern $(24 \mathrm{~cm} \times 24 \mathrm{~cm})$ placed on its back wall. The tube associated with the checkerboard pattern led to a dark reward box placed behind the back wall (not 
visible in figure $1 a$ ), containing a feeder with sucrose solution. Bees were trained to enter the apparatus and collect a reward of sugar water from the reward box.

The checkerboard associated with the reward was one of six checkerboards of different spatial frequencies and different orientations of contours (figure $1 b$ ), used in a semi-random succession throughout the training. Thus, the bees were attracted neither to a particular spatial frequency, nor to a particular orientation of contours, nor to a radiating pattern or a radially symmetrical one. The position of the rewarded compartment was altered at regular intervals by rotating the turntable on which the apparatus rested, so the bees could not rely on a particular location and were forced to look for the pattern. Over a period of several weeks, several groups of 6-10 bees, all individually marked, were trained in this way. Each rewarded visit of each bee was recorded.

Testing commenced after the bees had made about 50 rewarded visits to the apparatus. Prior to each test, the checkerboard pattern and the reward box were removed, and the tube leading to it was replaced by a fresh (unscented) one. This tube was closed from the back, similar to all of the other tubes. In the tests, a disc (24 $\mathrm{cm}$ in diameter) carrying a black-and-white pattern (exceptions will be stated) was attached to the back wall of each the 12 compartments. In the majority of the tests, four different patterns were used, each repeated three times, spread in regular alternation among the 12 compartments. In some of the tests, three patterns were used, each repeated four times. The criterion for a bee's choice was her crossing an imaginary line at the entrance to a compartment, at a distance of $30 \mathrm{~cm}$ from the pattern. The bees' entrances to the compartments were recorded by two experimenters simultaneously, each observing 6 of the 12 compartments for about ten minutes. The apparatus was rotated by $90^{\circ}$ after the first five minutes of the test. After the test, the training situation was restored and the bees were rewarded at least 10 times prior to the next test. Each test was conducted twice, the bees' choices being summed over the two tests.

The percentage of the bees' choices was calculated for each test pattern. Under random-choice conditions, i.e. when the bees express no preferences for any of the patterns, the choice probability of each pattern is $25 \%$ when four patterns are presented each in three compartments, and $33.3 \%$ when three patterns are presented each in four compartments. The distribution of choices among the patterns was compared with the random choice probability using the chi-square test.

\section{RESULTS AND CONGLUSIONS}

\section{(a) Tests with the checkerboard patterns}

Bees trained to the checkerboard patterns as described in Methods were first tested to examine whether they. have, indeed, learned the training patterns. In one test (figure $2 a$ ), the three patterns with square checkerboards were placed each in one compartment, separated by three compartments that displayed white discs. In a second test (figure $2 b$ ), the three patterns with the diagonal checkerboards were tested in the same way. In these tests, the results expected under random choice conditions are $8.3 \%$ for each checkerboard pattern, and $75 \%$ for the nine white compartments taken together. The results differ very significantly from this expectation. The bees clearly prefer the compartments containing a pattern over the blank compartments. However, the pattern of the lowest frequency was chosen more often than the others in both cases, despite the fact that all of the checkerboard patterns were rewarded equally often during the training. We shall return to this finding later on.

In figure $2 c$, we omitted the two high-frequency checkerboards and tested the other four patterns, with two blank compartments separating them from one another. Again, the preferences for the compartments containing a pattern was very significant, and the low frequency patterns were preferred over the high frequency ones.

In figure $2 d$, we tested four identical (this time circular) checkerboard patterns against four white and four grey discs, using the checkerboard that proved to be the most attractive pattern in figure $2 c$. The grey discs were composed of tiny black dots printed on white paper, taking care that black and white were at a ratio of $1: 1(50 \%$ white, $50 \%$ black $)$, as in the checkerboard patterns. The choice frequency for the checkerboard pattern is, again, significantly higher than the randomchoice probability. In addition, the white discs are preferred over the grey discs.

Three conclusions can be drawn from these results: (i) the bees have learned the patterns to which they have been trained; (ii) the bees prefer less disrupted checkerboards to more disrupted ones, i.e. they prefer low rather than high spatial frequencies; and (iii) even the most disrupted checkerboards are resolved by the bees (because, had these patterns appeared grey, the bees would have chosen the grey disc in figure $2 d$ as often as the checkerboard, or at least more often than the white disc, but they did not). It is important that the bees have learned the checkerboard patterns, because, in the subsequent tests, we shall test their preferences for patterns that do not resemble checkerboards.

\section{(b) Tests with different arrangements of bars}

In the tests shown in figure $3 a-d$, each of the four test patterns contained the same number $(12$ in $(a, b), 8$ in $(c, d))$ of black bars of equal size, but they differed in the arrangement of the bars. In figure $3 a$, the bees preferred the pattern with bars radiating from a circle in the centre of the disc over the one with bars radiating from a square, and the latter over a pattern with bars arranged on circles. The least attractive pattern in this test was the one in which the bars were arranged at random. A pattern containing radiating bars is also preferred over patterns containing bars of two perpendicular orientations, arranged in different ways (figure $3 b$ ). The results of figure $3 b$ show, in addition, that bees prefer a radially symmetrical pattern over patterns with a lower degree of symmetry. 
(a)
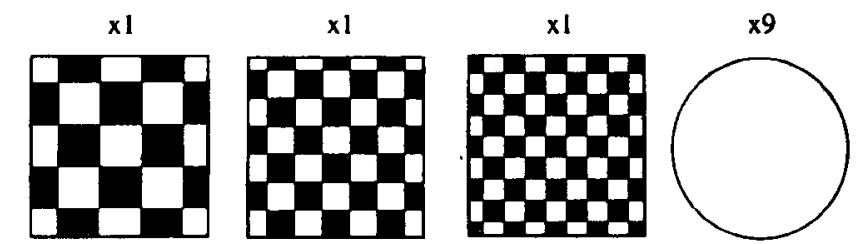

$n=223$

$p<10^{-4}$

$26.5 \%$

$22.9 \%$

$19.3 \%$

$31.4 \%$

(b)

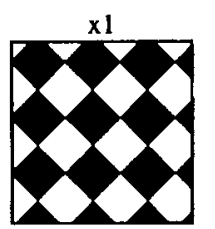

$20.1 \%$

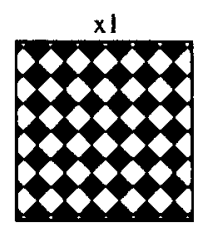

$19.6 \%$

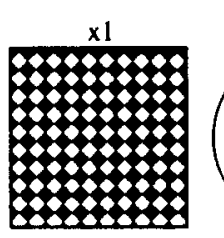

$12.1 \%$

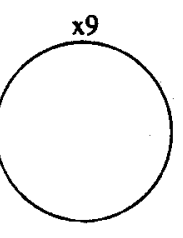

$n=209$

$p<10^{-4}$

(c)
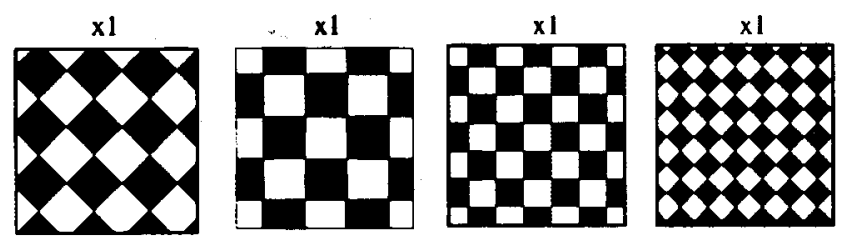

$48.3 \%$

$23.8 \%$

$21.0 \%$

$17.6 \%$

$13.8 \%$

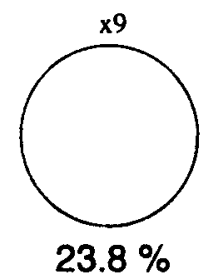

$n=548$

$p<10^{-4}$

(d)

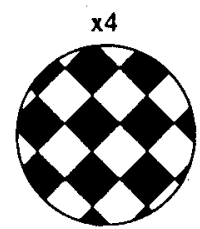

$55.2 \%$
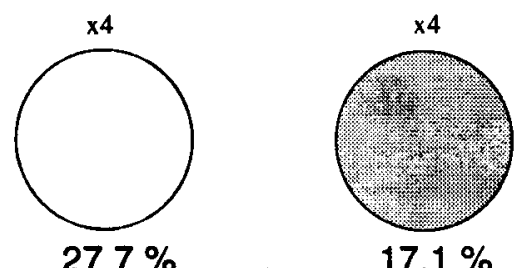

$17.1 \%$

$n=481$
$p<10^{-4}$

Figure 2. Results of tests examining the bees' learning performance. Here as well as in figures 3-9, $n$ denotes the total number of choices. The percentage of choices scored by each pattern is shown below the pattern. The $p$-values are results of chi-square tests comparing the test results with the random-choice probability, using absolute values of observed and expected choices. In $(a)$ and $(b)$, the three square and the three diagonal checkerboards, respectively, are tested against nine white discs. Random-choice probability is $8.3 \%$ for each checkerboard, and $75 \%$ for the nine white discs taken together. In $(c)$, the high-frequency patterns were omitted and the four other checkerboards were tested against eight white discs. The results show that the bees have learned the patterns to which they have been trained, and that low-frequency patterns are preferred over high-frequency ones. In (d), a low-frequency checkerboard pattern, presented in four different compartments, is tested against four white and four grey discs. Random-choice probability is $33.3 \%$ for each of the three patterns. The grey pattern is the least preferred one, showing that the high-frequency checkerboards (see $(a)$ and $(b)$ ) do not appear grey to the bees, i.e. they are resolvable.

In figure $3 c$, each of the four test patterns contained four radiating and four tangential bars, arranged in different ways. The bees preferred the pattern with eight planes of symmetry over all of the other patterns. However, the bilaterally symmetrical pattern (second from the left) and the asymmetrical pattern (third from the left), containing a group of three neighbouring radiating bars, were similarly attractive. This result suggests that the presence of a conspicuous group of radiating bars is at least as important as symmetry. The least attractive pattern in this experiment was the asymmetrical pattern (at the right) containing two pairs of radiating bars, separated by a tangential bar. In figure $3 d$, a radially symmetrical arrangement of eight radiating bars is successively transformed into a circular arrangement of the same bars by changing (from left to right) the orientation of two, four, or all of the bars. Although the pattern to the extreme left and the one to the extreme right are equally symmetrical, the pattern with radiating bars is much more attractive than the circular arrangement of the bars. The bees clearly distinguish well between these two types of pattern. Thus, the bees' spontaneous preferences are directed toward patterns containing radiating elements, as well as toward symmetrical patterns, but the former parameter is more important than the latter.

\section{(c) Tests with different numbers of bars}

In the next experiment, radial and circular bar patterns were examined separately, this time by varying the number of the bars within each set of four test patterns (figure $4 a, b$ ). The results show, first of all, that training to the checkerboards, in which the total amount of black area $(50 \%)$ was much larger than in the test patterns, did not result in a preference for the pattern that contained the largest total amount of black area. The pattern with the largest numiver of bars is not the most attractive one. Neither, ! owever, is the pattern with the smallest number of bars. In both cases, the bees prefer the pattern containing six bars. It is possible that the preference for six bars oriented in three directions separated by $60^{\circ}$ is related to the 
(a)

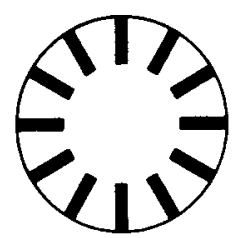

$32.4 \%$

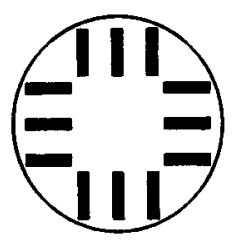

$26.6 \%$

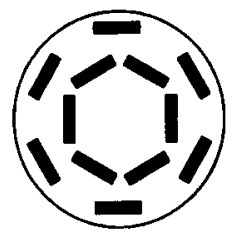

$22.9 \%$

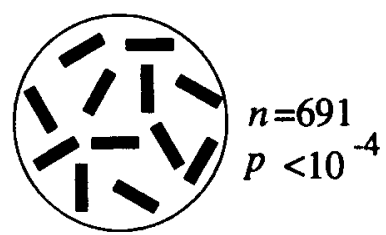

$18.1 \%$

(b)
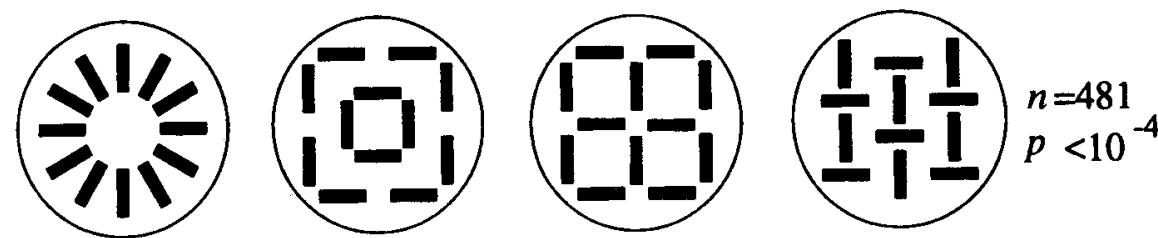

$32.2 \%$

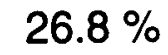

$24.9 \%$

$16.1 \%$

(c)
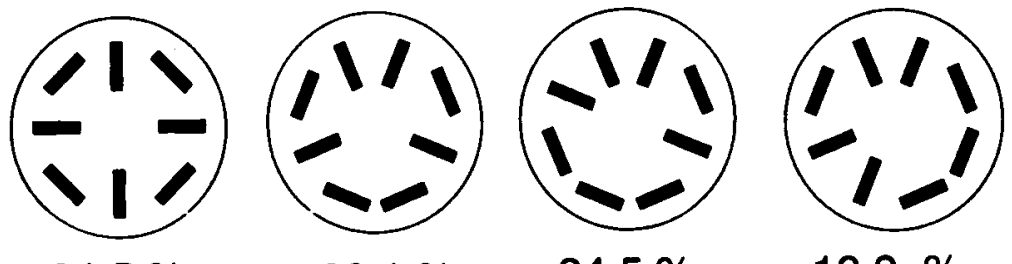

$n=931$
$p<10^{-4}$

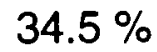

$22.1 \%$

$24.5 \%$

$18.9 \%$

(d)
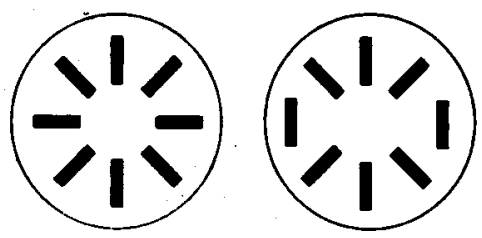

$22.8 \%$

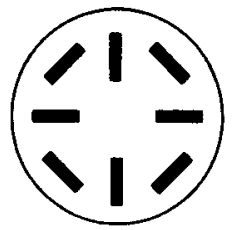

$22.1 \%$

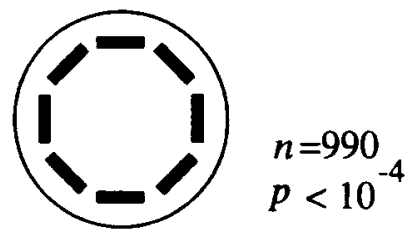

$22.4 \%$

Figure 3. (a-d) Tests with sets of four patterns (each presented in three compartments) containing equal numbers of equally-sized bars. Random-choice probability for each pattern is $25 \%$. The results reveal a preference for radiating bars and symmetrical arrangements of bars over random, circular and asymmetrical arrangements of bars.

(a)

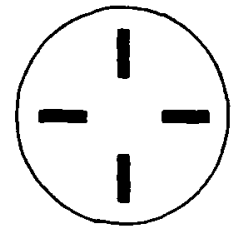

$22.2 \%$

(b)

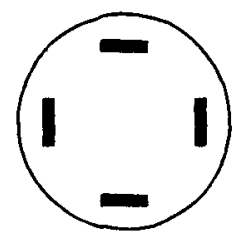

$26.7 \%$

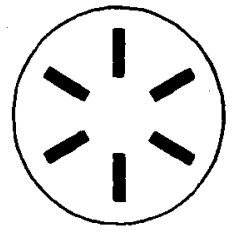

$32.9 \%$

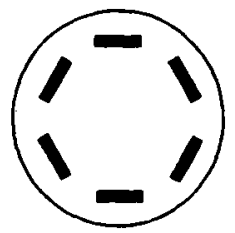

$31.2 \%$

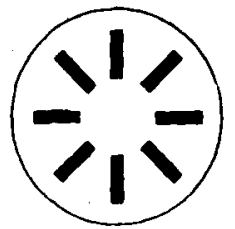

$21.4 \%$

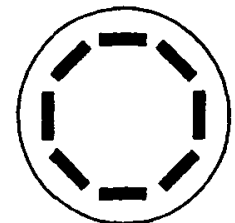

$21.4 \%$

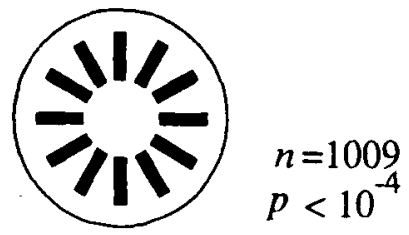

$23.5 \%$

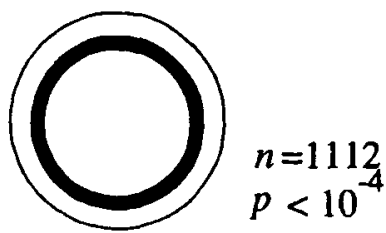

$20.7 \%$

Figure 4. $(a, b)$ Tests with sets of four patterns (each presented in three compartments) containing variable numbers of bars. Radiating $(a)$ and circular arrangements of bars $(b)$ are tested separately. In both cases, the bees prefer the pattern containing six bars. See text for comments.

hexagonal geometry of the bee's facetted eye. Based on a series of training experiments, Srinivasan et al. (1993) postulated the existence of three filters tuned to these three orientations. In the present context, however, it suffices to state that the bees' preferences include some subtle properties of the pattern that are independent of contour density. 
(a)
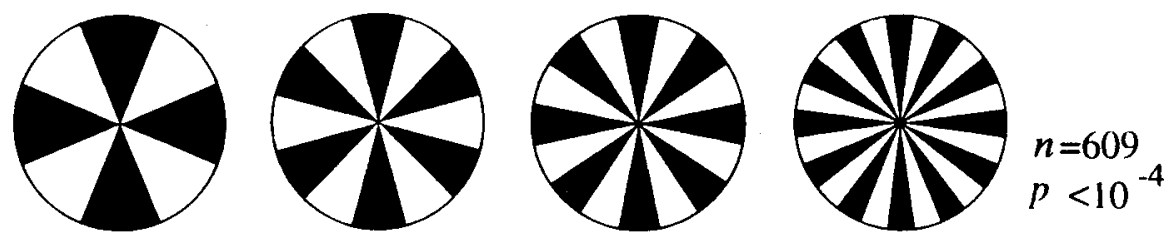

$35.5 \%$

$$
27.9 \%
$$

$15.6 \%$

$21.0 \%$

(b)
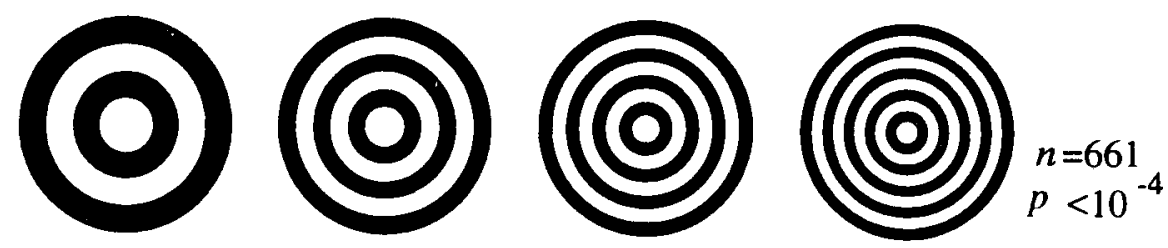

$39.9 \%$

$26.5 \%$

$20.7 \%$

$12.9 \%$
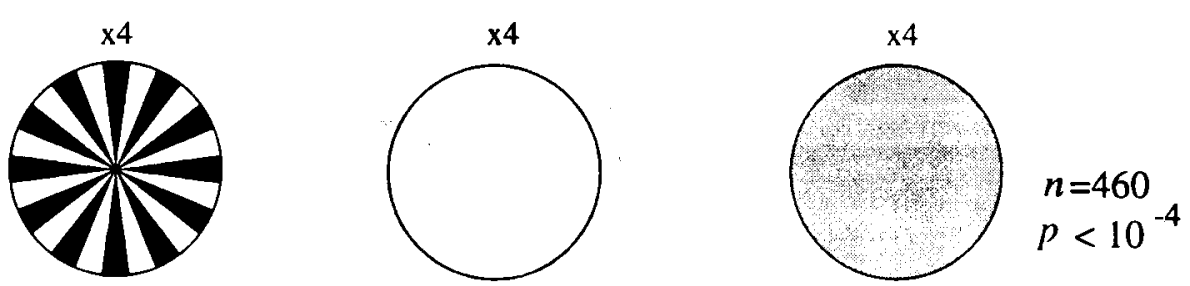

$62.4 \%$

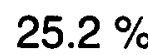

$12.4 \%$

(d)
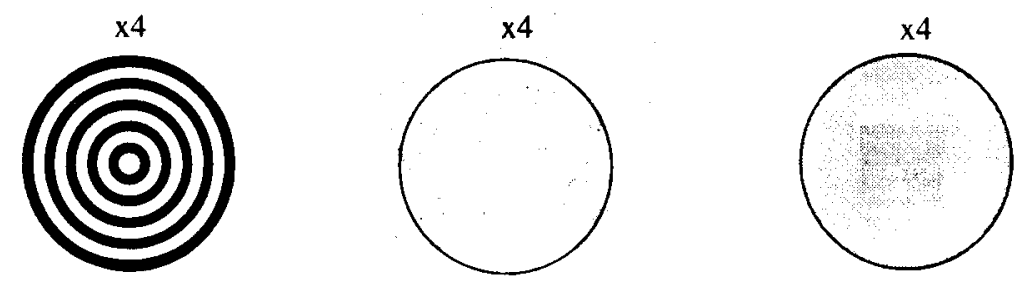

$n=429$

$p<10^{-4}$

$50.4 \%$

$30.5 \%$

$19.1 \%$

(e)
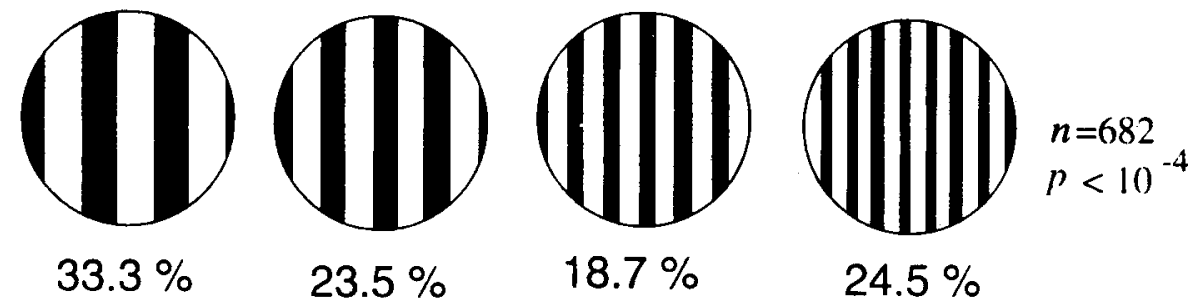

$18.7 \%$

$24.5 \%$

(f)
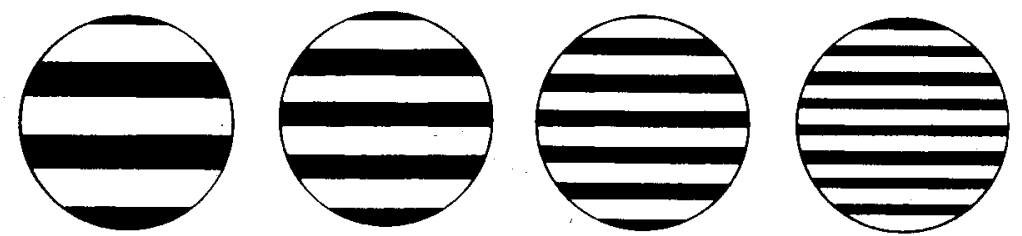

$n=741$

$36.7 \%$

$24.4 \%$

$21.5 \%$

$17.4 \%$

Figure 5. $(a, b)$ and $(e, f)$ : Tests with sets of four patterns (each presented in three compartments) that differ neither in type nor in the ratio of black and white areas, but rather in their spatial frequency. The bees consistently prefer low-frequency over high-frequency patterns (see also figure 2). In $(c)$ and $(d)$, the high frequency patterns used in $(a)$ and $(b)$, respectively, were tested against white and grey discs, each of the patterns presented in four compartments. The results show that the high-frequency patterns are resolvable to the bees.

\section{(d) Tests with patterns of different spatial} frequency

The patterns shown in figure $5 a, b$ were $50 \%$ black, as were the training patterns. In these two experiments, the patterns did not differ in type, but rather in their degree of disruption. In both cases, the most attractive pattern was the one with the lowest spatial frequency, as was already the case in the tests with the checkerboard patterns (see figure 2). This was certainly 
(a)

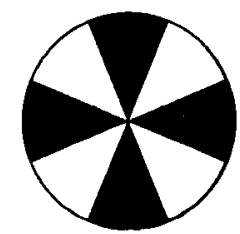

$37.8 \%$

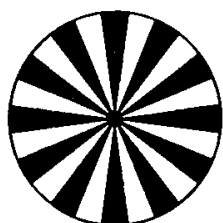

$41.9 \%$

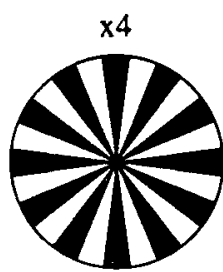

$59.5 \%$

(d)

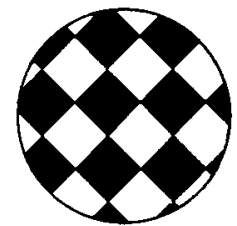

$37.1 \%$

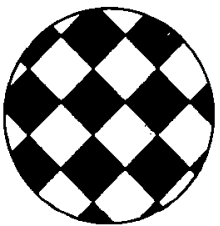

$26.8 \%$

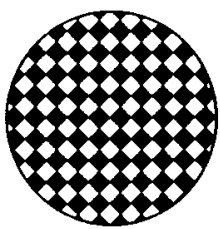

$21.6 \%$

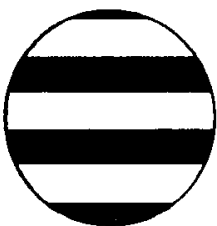

$21.4 \%$

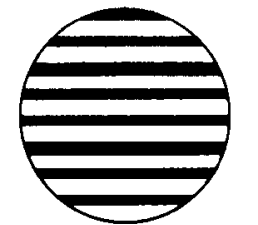

$22.1 \%$

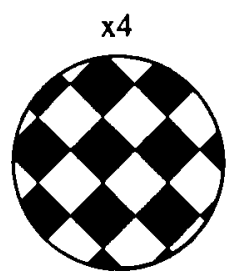

$21.3 \%$

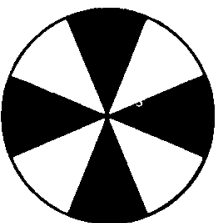

$28.7 \%$

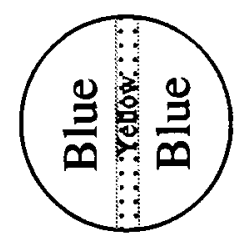

$16.0 \%$

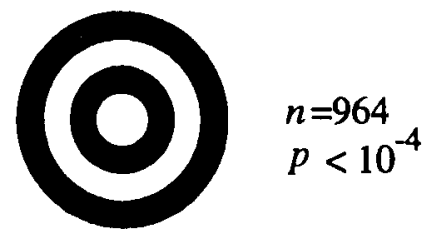

$14.0 \%$

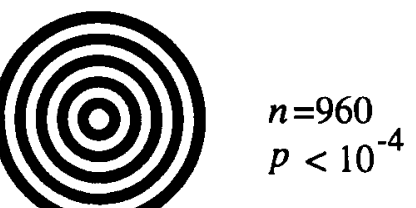

$14.4 \%$

$\mathrm{x} 4$

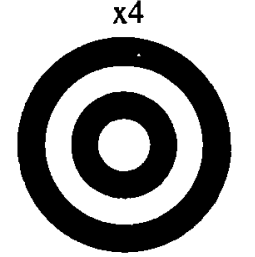

$n=691$

$p<10^{-4}$

Figure $6(a-d)$ Tests pitting different types of pattern against one another. In $(a)$ and $(b)$, four patterns were used, each presented in three compartments. In $(c)$, three patterns were used, each presented in four compartments. All of the patterns contain $50 \%$ black and $50 \%$ white areas. Radial sectors are preferred over all of the other types of pattern, irrespective of spatial frequency. In $(d)$, four patterns were offered, with two white compartments to either side of each. Two of the patterns were constructed of coloured papers as indicated. The rationale behind this test is explained the text.

not due to the bees' failure to resolve the finest patterns from the decision distance, because, when tested against homogeneous grey and white discs, these patterns were not confused with the grey discs (figure $5 c, d)$.

We obtained a clear preference for low-frequency patterns even when we used linear gratings, regardless of whether the contours were oriented horizontally or vertically (figure $5 e, f$ ). All of these results are in contrast to the earlier work (see $\S 1$ ) from which it was concluded that the bees' spontaneous preferences are directed toward high-frequency patterns. We shall return to this point in the Discussion.

\section{(e) Pitting different types of patterns against one another}

When the checkerboard patterns used during the training, and the three types of patterns used in figure 5 are pitted against one another, the radial grating is preferred over all of the other patterns (including the training pattern). This is regardless of whether the lowfrequency patterns (figure $6 a$ ), or the high-frequency patterns are used (figure $6 b$ ). We conclude that radiating sectors are innately attractive to the bees. In both cases, the least attractive pattern was the ring pattern. Even the radial grating with the finest sectors, which was not the most attractive pattern in figure $5 a$, was strongly preferred over the ring pattern that was the most attractive in figure $5 b$ (figure $6 c$ ). The bees seem to distinguish well between radiating sectors and ring patterns regardless of spatial frequency. The possibility that the ring pattern is least attractive due to training to the checkerboards must be dismissed, because, in a control experiment, we obtained similar results from bees trained to a black disc (not shown).

Although the bees have clearly learned the checkerboard pattern (see figure $2 d$ ), their preference for the sectored disc even in the presence of this pattern (figure $6 a-c$, see also figure $7 b, c$ below) might suggest that training was not as effective as we had hoped, i.e. the bees might have still retained some preferences learned during earlier experience with natural flowers. We therefore conducted another test, giving the bees a choice among four patterns, two of which were coloured (figure $6 d$ ). If training to the checkerboards 
(a)
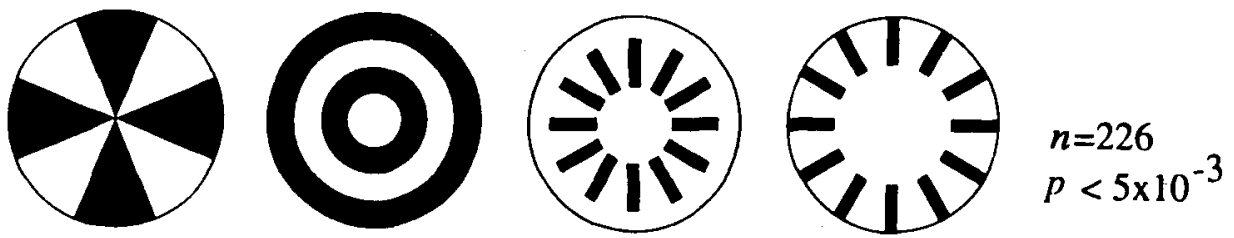

$35.4 \%$

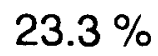

$21.3 \%$

$20.0 \%$

(b)
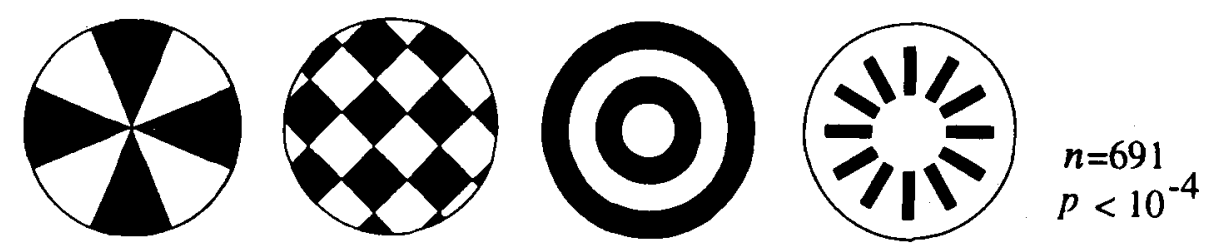

$36.4 \%$

$25.8 \%$

$19.7 \%$

$18.1 \%$

(c)
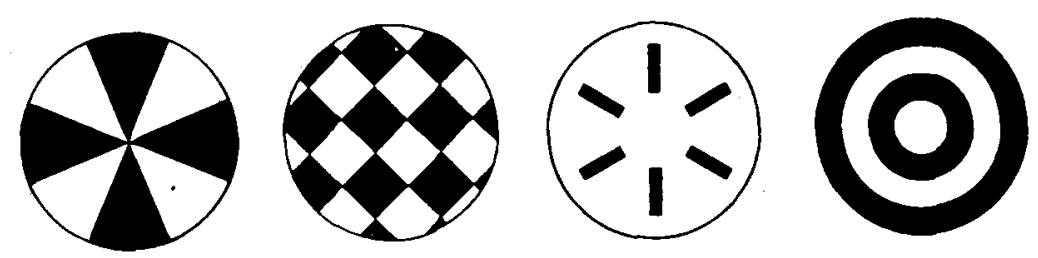

$n=1077$
$p<10^{-4}$

$37.1 \%$

$24.5 \%$

$20.7 \%$

$17.6 \%$

(d)
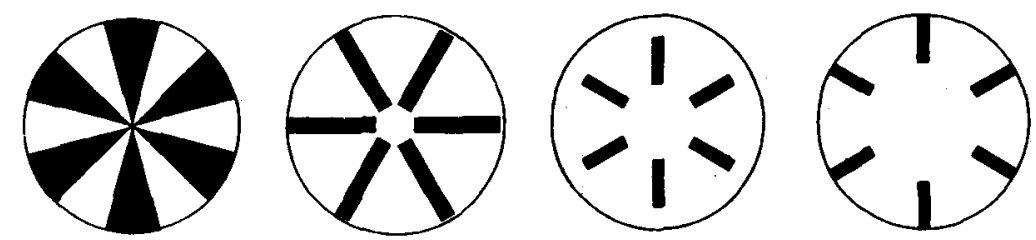

$n=960$

$38.0 \%$

$26.7 \%$

$14.9 \%$

$20.4 \%$

(e)

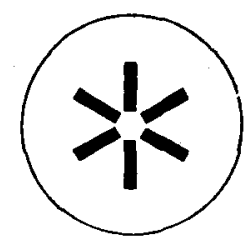

$20.4 \%$

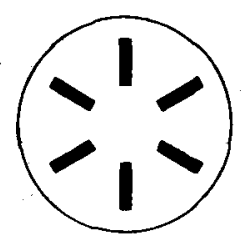

$28.8 \%$

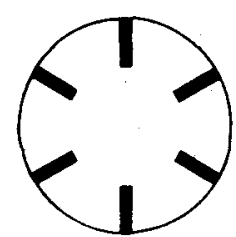

$28.8 \%$

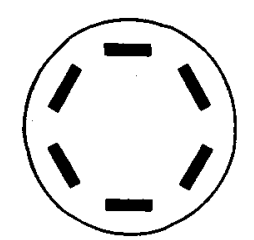

$n=838$

$p<10^{-4}$

$22.0 \%$

Figure 7. $(a-d)$ Tests with sets of four patterns (each presented in three compartments) differing in both the type of pattern and the ratio of black and white areas. The results show that radial sectors are more attractive than radiating bar patterns. In $(e)$, four different types of six-bar arrangements were pitted against one another. See text for comments.

had not extinguished previous preferences, then the bees would be expected to be attracted to the coloured patterns, because flower colour is a very effective cue. When bees are trained to a black-and-white striped rectangle and a coloured rectangle offered simultaneously, they prefer the coloured stimulus over the achromatic pattern when the two stimuli are pitted against each other (Collett 1994). In the test shown in figure $6 d$, however, the bees displayed no preference toward the coloured patterns; the best preferred pattern was now the checkerboard. This result suggests that training to the checkerboards has eliminated previously learned preferences.

From the experiments using bar patterns (see figure 3 ) we concluded that radiating bars are more attractive than any other arrangement of bars. From figure 6 we concluded that radiating sectors are more attractive than any other type of pattern containing $50 \%$ black areas. We now test the bees' choice behaviour when radial sectors and radiating bar patterns are presented simultaneously (figure $7 a-d$ ).

The results show that radial sectors are more attractive than radiating bar patterns. The patterns with 12 radiating bars, which were the most attractive ones in figure 3, are the least attractive in figures $7 a$, $b$. Even the pattern with six bars, that was the most attractive pattern in figure 4 , is less attractive than the radial sectors (figure $7 c$ ). Using a pattern with six sectors and three different types of radiating six-bars patterns (figure $7 d$ ), we again find a strong preference for the radial sectors (see also $\S 4$ ).

The results of figure $7 d$ lead to another interesting 
(a)

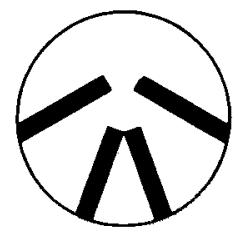

$30.2 \%$

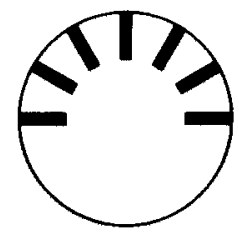

$31.4 \%$

(c)

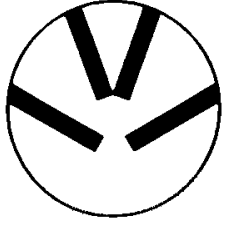

$27.3 \%$

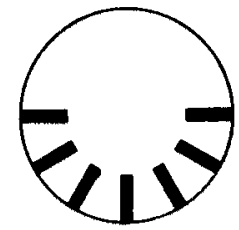

$28.6 \%$

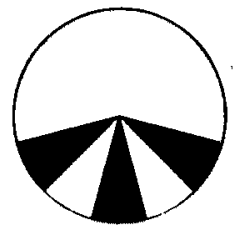

$30.7 \%$

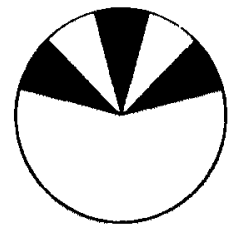

$26.8 \%$

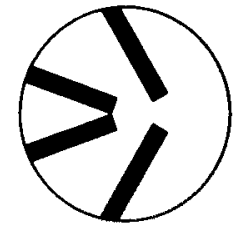

$23.0 \%$

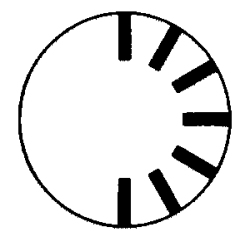

$21.5 \%$

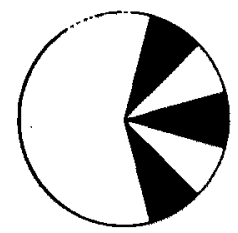

$24.0 \%$

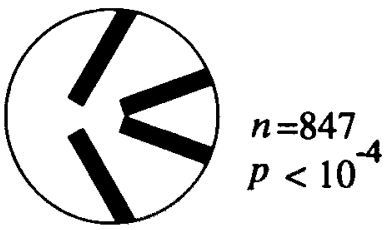

$19.5 \%$

Figure 8. $(a-c)$ Tests with sets of four patterns (each presented in three compartments), two of which display bilateral symmetry about a vertical line (dorsoventral bilateral symmetry), and two about a horizontal line (transversal bilateral symmetry). Random-choice probability for each pair is $50 \%$. Dorsoventral bilateral symmetry is preferred in all three cases.

finding: it seems that radiating bars are more attractive when they extend into the peripheral portions of the pattern than when they are placed nearer to the centre of the pattern (see also figure $9 b$ ). This is more specifically tested in figure $7 e$, using patterns with six equally-sized bars. We find that the radiating pattern placed at the centre of the figure is not as attractive as the two patterns in which the bars are placed farther away from the centre. In this test, the pattern with bars placed on a circle near the perimeter of the disc is not less attractive than the radiating pattern placed at the centre. This finding will be pursued in more detail in future experiments, and we shall not discuss it any further in the present paper.

\section{(f) Tests with bilaterally symmetrical patterns}

All of the results presented up to now suggest that the bee is innately attracted to patterns that resemble natural flowers in that they contain radiating elements, analogous to the petals of natural flowers. Radially symmetrical radiating patterns prove to be particularly attractive, but circular patterns, although equally symmetrical, are not attractive. Natural flowers, indeed, rarely possess round boundaries. However, not all natural flowers display radial symmetry. Many possess, instead, dorsoventral bilateral symmetry (i.e. symmetry about a vertical line). Dafni (1994) examined 981 flower species in the flora of South Africa and Israel with respect to their symmetry and found that $58 \%$ of the species are radially symmetrical, and $42 \%$ are bilaterally symmetrical.

To examine the bees' response to bilaterally symmetrical patterns, we now isolate the parameter 'radiating elements' from the parameter 'symmetry' by presenting the bees with four patterns containing the same number of radiating elements (figure $8 a-c$ ). In each of the three experiments, two of the patterns display a dorsoventral bilateral symmetry, and two a transversal symmetry. In all three cases, the bees prefer the former over the latter. Thus, again, the bees express an innate preference towards flower-like patterns.

\section{(g) Tests with clustered arrangements of bars}

It is trivial to assume that the global features of a pattern viewed from a distance can only be detected when the pattern is large enough to allow the resolution of such features. To overcome this difficulty, many plants have evolved, instead of large flowers, inflorescences consisting of many small flowers arranged in clusters to attract pollinators even from a distance. If the bees' preference is indeed related to the appearance of natural flowers, as we have proposed above, then we would expect the bees to prefer clusters of small elements over an unclustered arrangement of the same elements. In addition, we would expect the bees to prefer a larger number of clusters over a smaller one. The next set of tests was designed to test these expectations.

We now trained bees to a single small horizontal bar (figure $9 a$ ), rather than to the checkerboard patterns used before. Training to a single small element acts against a preference for a cluster composed of several small elements that we intend to offer in the tests, as well as against the preference for a large number of clusters.

In figure $9 b$, all of the test patterns had six bars radiating from the centre of the disc. The four patterns differ in the size (length and width) of the bars. The 
(a) Train

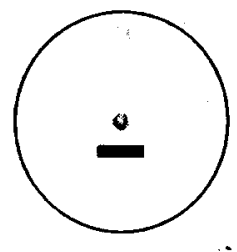

(b)

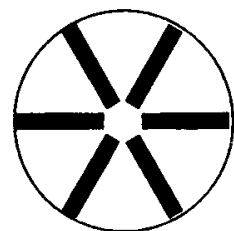

$29.9 \%$

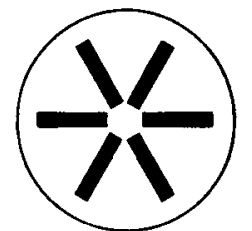

$26.7 \%$

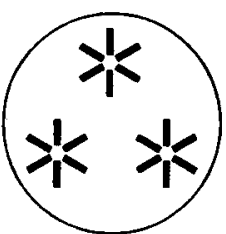

$28.8 \%$

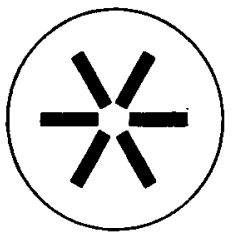

$21.6 \%$

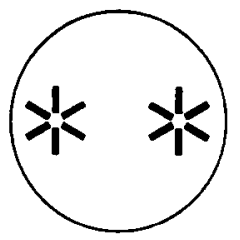

$19.9 \%$

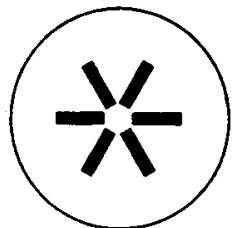

$n=931$
$p<10^{-4}$

$22.5 \%$

(c)

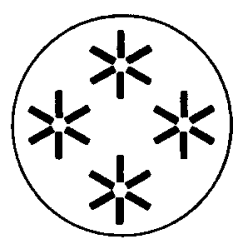

$29.1 \%$

(d)
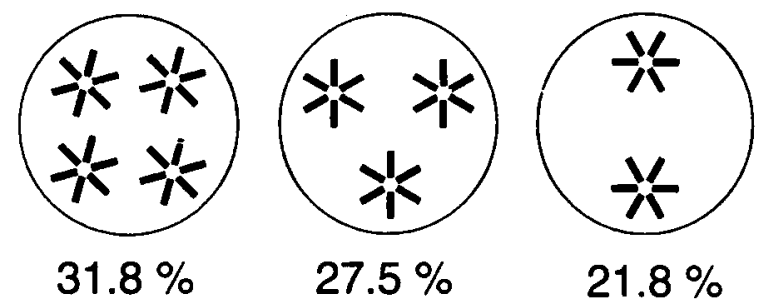

(e)

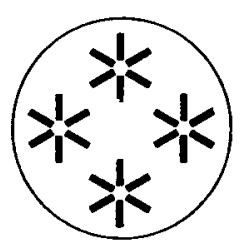

$36.9 \%$
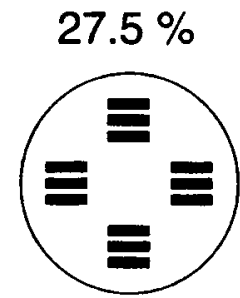

$23.7 \%$

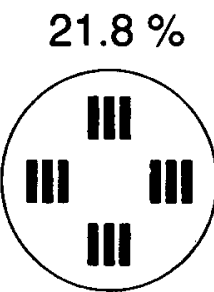

$19.6 \%$

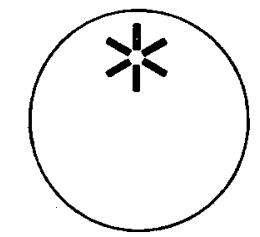

$n=691$
$p<10^{-4}$

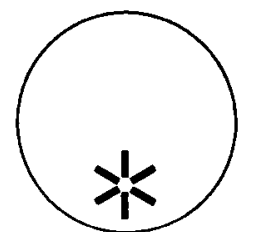

$n=481$

$p<10^{-4}$

$18.9 \%$

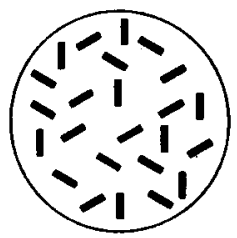

$n=990$

$19.8 \%$

Figure 9. Bees trained to a single small horizontal bar $(a)$ were tested with clusters of bars $(b-e)$. The results show that large patterns are more attractive than smaller ones $(b)$, and that a greater number of clusters is more attractive than a small number $(c, d)$. The shape of the clusters is important as well: $(e)$ the bees prefer clusters of radiating bars over ones with parallel bars, or with a random arrangement of bars.

results show that the pattern is the more attractive the larger it is, despite the fact that the bees were trained to a small bar.

We proceed by using flower-like clusters of six bars, each being a miniature reproduction of the pattern shown in figure $9 b$. When given a choice among one, two, three and four such 'flowers', the bees prefer targets with three or four over such with only one or two, regardless of the arrangement of the flowers within the disc (figure $9 c, d$ ). In a further test (figure $9 e)$, the pattern with four flowers was strongly preferred over a pattern with the same number (24) of equallysized single bars arranged randomly (using the same three orientations of bars as in the flower pattern), as well as over four clusters composed of parallel bars, with the bars running either horizontally or vertically. The total amount of black areas was the same in all of the four patterns used in this test. (The preference for the horizontal bars over the vertical ones is certainly a consequence of training the bees to a horizontal bar, see figure $9 a$.) This experiment demonstrates, again, the bees' preference for radiating elements.

We conclude from the results of figure 9 that: (i) clusters of elements are more attractive than an unclustered arrangement of the same elements; (ii) a large number of clusters is more attractive than a small number of clusters; and (iii) the global shape of single clusters plays a role in the bees' preferences. Thus, again, bees prefer flower-like pattern parameters.

\section{(h) Preferences at a distance versus preferences at a close range}

The flicker theory formulated by Exner (1876) was supported by data from landing bees (see $\S 1$ ), without testing whether or not the discrimination parameters 
(a)

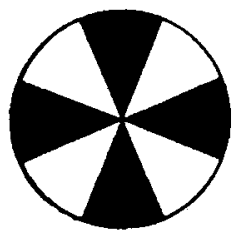

entrances: 84 landings: 0 touchings: 0

(b)

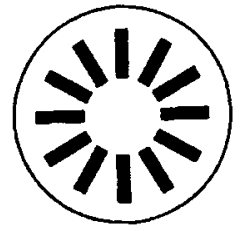

entrances: 127 landings: 0 touchings: 5

(c)

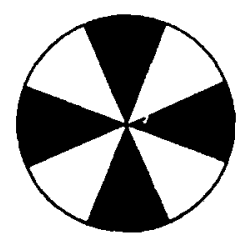

entrances: 91 landings: 2 touchings: 15

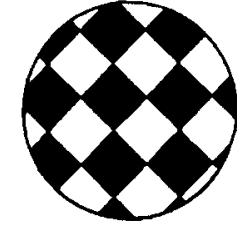

entrances: 58 landings: 44 touchings: 18

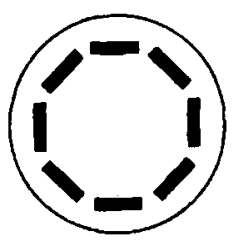

entrances: 82 landings: 1 touchings: 0

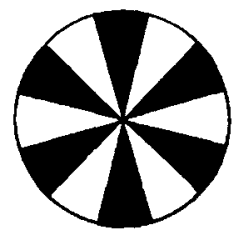

entrances: 96 landings: 2 touchings: 13

Figure 10. $(a-c)$ Dual-choice tests after training bees to the checkerboard patterns. The two test patterns are separated by five white compartments on each side. Three different choice criteria are used simultaneously: (i) entrances to the compartment, as in figures 2-9; (ii) touching the tube in the centre of the pattern (see \$2); and (iii) landing on the tube. The bees readily enter the compartments in all cases, but they hardly ever land on the tube associated with a novel pattern. Landings occur almost exclusively on the training pattern $(a)$. See text for comments.

depend upon the bee's distance from the pattern. Before we criticize the earlier workers for having neglected this aspect, however, we must ask whether the discrepancies between their results and ours are indeed a consequence of the different choice criteria used.

During training, our bees have learned that the access to the reward is through the tube in the centre of the pattern; they must land on that tube prior to each reward. They are, therefore, bound to have learned not only the global features of the checkerboard as viewed from a distance, and as they approach, but, in addition, its local features as viewed at a very close range. The test patterns, on the other hand, are novel to the bees with respect to both global and local

parameters. We now ask what would have been our bees' responses to the test patterns had we measured their decisions at a very close distance from the patterns, rather than at the threshold to the compartments.

We investigated this question by conducting tests in which we differentiated among three choice criteria by simultaneously recording: (i) the bees' entrances to the compartments; (ii) their landings on the tube in the centre of the pattern (see §2); and (iii) their touching that tube. Since it was impossible, without using several video cameras, to record these three types of decisions in all of the 12 compartments simultaneously, we presented the bees with only two patterns at a time, separated, on each side, by five compartments displaying plain white discs. We conducted three such experiments, the results of which are shown in figure $10 a-c$. Although the proportions of the entrances are in general agreement with our earlier results, touchings and landings occur almost exclusively at the tube associated with the checkerboard pattern to which the bees have been trained (figure 10a). The other (novel) patterns are rarely approached close enough for the bees to touch or land on the tube, as can be visualized in figure 11 that shows flight trajectories of four bees (one in figure $11 a$, three in figure $11 b$ ) during two different tests, each using 12 patterns that are different from the training patterns. The drawings are not based on filming the bees, because the design of the apparatus made filming impossible. Instead, we show trajectories in which the bees were flying slowly enough to make it possible to record their flight by eye. As soon as the bee has approached the test pattern, she recognizes it as being different from the training pattern. The bee then retreats immediately to try again in another compartment.

From the results of figures 10 and 11 it is clear that bees select different pattern parameters depending on the distance from which they view the patterns. Zhang et al. (1992) have provided further evidence, based on the results of training experiments, to support the idea that bees differ in their responses to pattern parameters depending on whether they are required to make their choices at a close or a farther range. Since, however, all of our test results are based on choices done at a distance, close-range cues learned during training could not have been used by the bees for making their decisions.

\section{DISGUSSION}

The present study constitutes the first investigation of flying bees' spontaneous pattern preferences towards patterns presented on vertical planes, and with the criterion a decision at some distance from the patterns. Our results differ from those of earlier work in which patterns were placed on a horizontal plane and with landings as the criterion.

At first sight, it would seem that the main difference between the earlier results (see $\$ 1$ ) and ours lies in the finding that our bees prefer less disrupted patterns over more disrupted ones. However, we do not consider this discrepancy to be the main result of our experiments. 
(a)

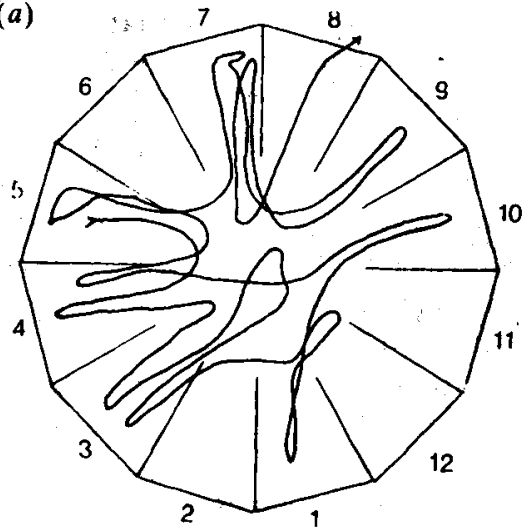

(b)

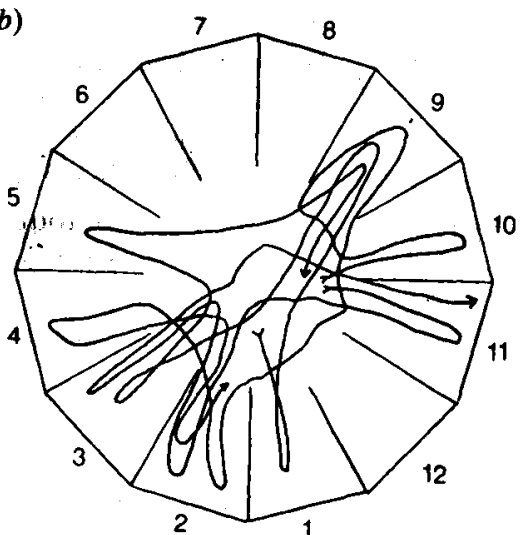

Figure 11. Flight trajectories of individual bees during two tests $(a, b)$ with patterns that are not identical with the checkerboard patterns used during the training. The bees' flight behaviour reveals that they use different cues depending on whether they view the pattern at a distance or at a near range. See text for further comments.

When viewed from some distance, low frequency patterns produce higher contrasts than do high frequency patterns, a problem that disappears when the patterns are viewed at a close range. Thus, in our experiments, although the high frequency patterns can be resolved (see figure 5), the global spatial parameters of the patterns are probably more obvious when the pattern has coarse features than when it has fine features. The bees' preference for radial sectors over radial arrangements of bars (see figure 7) can; again, be explained by the greater salience of the former: the total amount of black area is larger in the sectors than in the bars. It has been shown several times that black patterns on a white ground are more effective than white patterns on a black ground (Schnetter 1968; Wehner $1972 b$ ). Thus, larger areas of black would tend to make global parameters more obvious. In addition (see figure 2 and figure $5 a, b, e, f$, it is only when the global parameter is kept constant among the test patterns that the bees prefer the low frequency patterns. When different types of global parameters are compared with one another, the degree of disruption is not important (see figures 3, 4 and 6-8).

Thus, our main conclusions involve the use of global spatial parameters whose attractiveness is independent of spatial frequency. The earlier workers, using landings as a choice criterium, were unable to discover such parameters because their bees had little more than temporal cues (i.e. flicker frequency) to rely upon. In spontaneous preference tests conducted by Hertz (1930), the bees preferred fine checkerboard patterns over a variety of other shapes, including such with radiating elements.

\section{(a) The hierarchy of global pattern parameters in the bees' spontaneous choice behaviour}

Under our experimental conditions, bees are not attracted to a high-frequency contrast generated at the eye, but rather to the particular global appearance of the pattern. The finding that random arrangements of elements are not attractive (see figure $3 a$ and figure $9 e$ ) again suggests that the bees' spontaneous preferences are directed toward well-defined global spatial properties of the pattern.
The preference for radial (see figure 3), as well as dorsoventral bilateral symmetry (see figure 8 ), could not be demonstrated in the earlier studies, because symmetry is a global parameter that can only be perceived at some distance from the pattern. It should be noted, however, that radially symmetrical patterns have often been used in the earlier studies (see reviews by von Frisch 1965; Wehner 1981), although the earlier workers never set out to investigate systematically the role that symmetry might play in the bees' pattern preferences.

We find that, despite the bees' obvious preference for symmetrical patterns, several further global parameters are at least as important. The two global types of pattern toward which the bees express a clear positive or negative preference, respectively, are radial patterns on the one hand, and circular patterns on the other hand (see figures $3 a, d, 6 a-c$ and $7 a-c$ ), despite the fact that both of these types of pattern are highly symmetrical. The presence of radiating elements is more important than symmetry (see figure $3 d$ ). It is only when the number of radiating elements does not differ among the test patterns that symmetry becomes important (see figure $3 c$ and figure 8 ).

Further global spatial features prove to be important as well. Radial sectors (with $50 \%$ total black area) are more attractive than radiating bar patterns (with less than $50 \%$ total black area, see figure 7). Large patterns are more attractive than small ones (see figure $9 b$ ), and patterns containing clustered elements are more attractive when the number of clusters is large than when it is small (see figure $9 c, d$ ).

\section{(b) The role of image motion}

It was shown in an earlier study that freely flying bees strive to avoid image motion when they are required to discriminate between patterns containing different orientations of contours (Lehrer et al. 1985). It was also shown that image motion is not necessary in a pattern discrimination task (Srinivasan et al. 1993). The possibility that our bees spontaneously preferred radial sectors and radially symmetrical arrangements of radiating bars (see figures $3 a, b, d$ and $6 a-c$ ) simply because these are the patterns whose contours produce 
the smallest amount of image motion at the flying bee's eye, and thus remain rather constant in their appearance, must be excluded in the light of the results shown in figures $8 a-c$ and $9 b, c$. In all of these tests, the bees' preferences cannot be explained by arguing that the most attractive patterns are those whose contours produce the smallest or the largest amount of image motion on the flying bee's eye.

\section{(c) Spontaneous preference for flower-like patterns}

Our results can be summarized by saying that bees innately prefer patterns displaying conspicuous global flower-like shape parameters.

Our finding that bees are innately attracted to patterns that resemble natural flowers is closely related to the modern theory of adaptive (or selective) learning, suggesting that small-brain animals possess so-called learning predispositions (Lauer \& Lindauer 1971), and are thus programmed, or prepared, to learn cues that are relevant to their needs, but not cues that are irrelevant (Gould 1982; Menzel 1985). A strong argument for this theory is the finding that there are several stimuli that bees are clearly unable to associate with a food reward, although they perceive them and react to them in other behavioural contexts. For example, bees cannot be trained to discriminate between a flickering light of a particular colour and a steady light of the same colour presented at the feeding place (Srinivasan \& Lehrer 1984), despite the fact that they clearly perceive flicker, as demonstrated by their spontaneous preference for high-frequency flicker (Wolf 1933), as well as by the strong, phasic responses of the photoreceptors to flickering stimuli (Autrum \& Stöcker 1950). Further, bees are very poor at learning a particular orientation of the magnetic field present at the feeding site (Walker \& Bitterman 1989), although they clearly react to magnetic stimuli in the contexts of comb building (Lindauer \& Martin 1968) and dance communication (Martin \& Lindauer 1973). Similarly, while bees use sounds in the context of dance communication (Esch 1964; Michelsen et al. 1986), their performance in associating sounds with a food reward is rather poor (Towne \& Kirchner 1989). Finally, in the context of navigation, bees use directional information derived from the polarization pattern of the sky (von Frisch 1965), but they cannot be trained to a particular E-vector orientation when it is used to mark the food source (Lau 1976). Natural flowers, indeed, attract pollinators neither by making noise, nor by flickering, nor by using magnetic fields or particular $E$ vectors. All of these findings suggest that some 'knowledge' on what is and what is not relevant in a particular behavioural context is innate to the bee. In the foraging task, the bees spontaneously prefer flower-like shape parameters.

Although we have looked at pattern preferences only in black-and-white, it should by no means be forgotten that colour is another strong cue for attracting pollinators. It would be far beyond the scope of this paper to cite the innumerable studies that have proved this. Colours are mainly important in the task of discriminating among different flowers. Given the large variety of colours that flowers have evolved, and the fact that bees forage at different flower species depending on the season, it is unlikely that bees possess an innate preference for particular colours. The typical shape parameters of flowers, on the other hand, are only few, namely size, the number of petals, the presence of radiating elements, and radial or bilateral symmetry. We have shown that bees are innately attracted to these properties and reject random patterns, as well as non-radiating and assymmetrical patterns. Our results suggest that flower shape acts together with colour as a cue for the bee to identify natural food sources.

Very recent studies have proposed simple mechanisms by which two-dimensional spatial parameters could be recognized in a neurally economical way. Even colour discrimination is accomplished with the help of only three types of broad-band filters, each tuned to a particular portion of the spectrum. Based on training experiments, Srinivasan et al. (1993) proposed the existence of filters tuned to three particular orientations of contours. It is possible that bees possess, in addition, filters tuned to radiating and circular shape elements. Such filters would enable the bee to use global parameters to discriminate numerous patterns with only a small number of specialized neurons.

M.L. and R.G. thank Adrian Horridge and Mandyam Srinivasan for the kind invitation and generous hospitality. The study was partly supported by funds from the Fujitsu Corporation of Japan to Adrian Horridge and Mandyam Srinivasan, and an Australian Commonwealth Science and Technology Grant for Bilateral Collaboration to Mandyam Srinivasan.

\section{REFERENCES}

Anderson, A.M. 1977 Parameters determining the attractiveness of stripe patterns in the honey bee. Anim. Behav. 25, 80-87.

Autrum, H. \& Stöcker, M. 1950 Die Verschmelzungsfrequenzen des Bienenauges. Z. Naturf. 5b, 38-43.

Campan, R. \& Lacoste, G. 1971 Les préférences visuelles spontanées chez le grillon Nemobius sylvestris et leur modification sous l'effect de l'experience. Cong. Soc. Sav. Toulouse 96, 465-483.

Collett, T.S. 1994 Do bees bind the components of panoramic patterns? Proc. 12th Congress of the International Union for the Study of Social Insects (IUSSI). Pub. Université Paris Nord, p. 93.

Cruse, H. 1972 Versuch einer quantitativen Beschreibung des Formensehens der Honigbiene. Kybernetik 11, 185-200.

Dafni, A. 1991 The reaction of Amphicoma beetles to red models and its implications in pollination. Proceedings of plant reproductive ecology: progress and perspectives, p. 18. Uppsala.

Dafni, A. 1994 A note on floral symmetry, nectar guides, and the bee's scanning behaviour. Funct. Ecol. (In the press.)

Esch, H. 1964 Beiträge zum Problem der Entfernungsmessung in den Schwänzeltänzen der Honigbiene. $Z$. vergl. Physiol. 48, 534-546.

Exner, S. 1876 Über das Sehen von Bewegung und die Theorie des zusammengesetzten Auges. Sber. Kaiserl. Akad. Wiss., Matt.-Nat. Wiss. C1 72, 156-191. 
Free, J.B. 1970 Effect of flower shapes and nectar guides on the behaviour of foraging bees. Behaviour 37, 269-285.

Frisch, K. von 1915 Der Farbensinn und Formensinn der Bienen. Zool. Jb. Abt. allg. Zool. Physiol. 35, 1-182.

Frisch, K. von 1965 Tanzsprache und Orientierung der Bienen. Springer: Berlin Heidelberg New York.

Gould, J.L. 1982 Ethology: the mechanisms of evolution and behavior. New York: Norton.

Gould, J.L. 1986 Pattern learning by honey bees. Anim. Behav. 35, 990-997.

Hateren, H.J. van, Srinivasan, M.V. \& Wait, P.B. 1990 Pattern recognition in bees: orientation discrimination. $J$. comp. Physiol. B 167, 649-654.

Hertz, M. 1930 Die Organisation des optischen Feldes bei der Biene II. Z. vergl. Physiol. 11, 107-145.

Hoefer, I. \& Lindauer M. 1976 Der Schatten als Hilfsmarke bei der Orientierung der Honigbiene. J. comp. Physiol. 112, 5-18.

Lau, D. 1976 Reaktionen von Honigbienen (Apis mellifica L.) auf Polarisationsmuster an der Futterquelle. Zool. Garten, Neue Folge, Jena 46, 1/2, 34-38.

Lauer, J. \& Lindauer, M. 1971 Genetisch fixierte Lerndispositionen bei der Honigbiene. Akad. Wiss. Lit., Mainz, Inf. Org. 1, 1-87. .-

Lehrer, M., Wehner, R. \& Srinivasan, M.V. 1985 Visual scanning behaviour in honeybees. J. comp. Physiol. A 157, 405-415.

Lehrer, M., Srinivasan, M.V., Zhang, S.W. \& Horridge, G.A. 1988 Motion cues provide the bee's visual world with a third dimension. Nature, Lond. 332, 356-357.

Lindauer, M. \& Martin, H. 1968 Die Schwereorientierung der Bienen unter dem Einfluss des Erdmagnetfeldes. $Z$. vergl. Physiol. 60, 219-243.

Manning, A. 1957 Some evolutionary aspects of the flower constancy of bees. Proc. R. Phys. Soc. 25, 67-71.

Martin, H. \& Lindauer, M. 1973 Orientierung im Erdmagnetfeld. Fortschr. Zool. 21, 211-226.

Menzel, R. 1969 Das Gedächtnis der Honigbienen für Spektralfarben. II. Umlernen und Mehrfachlernen. $Z$. vergl. Physiol. 63, 290-309.

Menzel, R. 1985 Learning in honey bee in an ecological and behavioural context. In Experimental and behavioural ecology (ed. M. Lindauer \& M.B. Hölldobler)(Fort. Zool. 31), pp. 55-74. Stuttgart: Fischer.

Menzel, R. 1990 Learning, memory and 'cognition' in honeybees. In Neurobiology of comparative cognition (ed. R.P. Kesner \& D.S. Olton), pp. 237-292. Hillsdale NJ: Erlbaum.

Michelsen, A., Kirchner, W.H. \& Lindauer, M. 1986 Sound and vibration signals in the dance language of the honeybee, Apis mellifera. Behav. Ecol. Sociobiol. 18, 207-212.
Reichardt, W. 1973 Musterindizierte Flugorientierung. Versuche an der Fliege Musca domestica. Naturwissenschaften 60, 122-138.

Schnetter, B. 1968 Visuelle Formunterscheidung der Honigbiene im Bereich von Vier- und Sechstrahlsternen. Z. vergl. Physiol. 59, 90-109.

Srinivasan. M.V. \& Lehrer, M. 1984 Temporal acuity of honeybee vision: behavioural studies using flickering stimuli. Physiol. Entomol. 9, 447-457.

Srinivasan. M.V. \& Lehrer, M. 1988 Spatial acuity of honeybee vision and its chromatic properties. J. comp. Physiol. A 162, 159-172.

Srinivasan, M.V., Lehrer, M., Zhang, S.W. \& Horridge,G.A. 1989 How honeybees measure their distance from objects of unknown sizes. J. comp. Physiol. A 165, 605-613.

Srinivasan, M.V., Zhang, S.W. \& Rolfe, B. 1993 Is pattern vision in insects mediated by 'cortical' processing? Nature, Lond. 362, 539-540.

Srinivasan, M.V., Zhang, S.W. \& Whitney, K. 1994 Visual discrimination of pattern orientation by honeybees. Phil. Trans. R. Soc. Lond. B 343, 199-210.

Towne, W.F. \& Kirchner, W.H. 1989 Hearing in honeybees: detection of air-particle oscillations. Science, Wash. 244, 686-688.

Varjú, D. 1976 Visual edge fixation and negative phototaxis in the mealworm beetle Tenebrio molitor. Biol. Cyber. 25, 17-26.

Walker, M.M. \& Bitterman, M.E. 1989 Attached magnets impair magnetic field discrimination by honeybee. $J$. exp. Biol. 141, 447-451.

Wehner, R. $1972 a$ Dorsoventral asymmetry in the visual field of the bee, Apis mellifera. J. comp. Physiol. 77, 256-277.

Wehner, R. $1972 b$ Pattern modulation and pattern detection in the visual system of Hymenoptera. In Information processing in the visual systems of Arthropods (ed. R. Wehner), pp. 183-194. Berlin, Heidelberg, New York: Springer.

Wehner, R. 1981 Spatial vision in arthropods. In Handbook of sensory physiology VII/6C (ed. H. Autrum), pp. 287-616. Berlin, Heidelberg, New York: Springer.

Wehner, R., Lindauer, M. 1966 Die optische Orientierung der Honigbiene (Apis mellifica) nach der Winkelrichtung frontal gebotener Streifenmuster. Zool. Anz. Suppl. 30, 239-246.

Wolf E. 1933 Das Verhalten der Bienen gegnüber flimmernden Feldern und bewegten Objekten. $Z$. vergl. Physiol. 20, 151-161.

Zerrahn G. 1934 Formdressur und Formunterscheidung ber der.Honigbiene. Z. vergl. Physiol. 20, 117-150.

Zhang, S.W., Srinivasan, M.V. \& Horridge, G.A. 1992 Pattern recognition in honeybees: local and global analysis. Proc. R. Soc. Lond. B 248, 55-61.

Received 5 May 1994; accepted 12 August 1994 